\title{
IMAGE ANALYSIS OF CRACKS IN CONCRETE: METHODOLOGY, OPPORTUNITIES AND PITFALLS
}

\author{
P. STROEVEN \& H. HE \\ Faculty of Civil Engineering and Geosciences, Delft University of Technology, The Netherlands.
}

\begin{abstract}
Damage in concrete is mostly visualised in sections or at the surface of specimens subjected to internal and/or external loading. It continuously develops by growth and coalescence of tiny cracks into a spatial network structure. This structure can be seen as the finger-print of the material reflecting its history of loadings under given environmental conditions. The methodology of contrast improvement as an essential link in visualising damage is touched upon. However, a major focus of the paper is on describing damage by submitting images of crack patterns consisting of lineal features in the plane to a sweeping test line system and counting intersections. To obtain three-dimensional damage information in an economic way, the damage structure is assumed in the most general case revealing a partial orientation of mixed lineal and planar nature (the so-called 'Stroevenconcept'). The practical cases are elaborated of prevailing compressive and tensile stresses. This reduces the number of unknown crack portions to two. As a consequence, quantitatively analyzing the image patterns can be restricted to vertical sections only. This involves a dramatic reduction of sawing efforts and simplifies the image analysis stage as well, of course. Only two orthogonal intersection counting operations are required for the assessment of specific crack surface area and of the degree and direction of preferred crack orientation. When observations would have been obtained in more directions, so-called roses of intersections (or intersection densities) can be determined. For very large images this would be circles. For random cracks in the image plane a circle around the origin, for oriented cracks, a circle through the origin is found. This concept, in addition to mathematical formulations is employed to demonstrate that automation of quantitative image analysis generally yields biased information, unless the analysis is executed under conditions discussed herein.

Keywords: analog and digitised images, automation of image analysis, concrete, cracking, line scanning, rose of intersections.
\end{abstract}

\section{INTRODUCTION}

Concretes are undergoing a process of degradation during life time of the engineering structure. This process of gradual failure was already recognised by Brandzaeg in 1927 [1]. The 3D network structure of growing and coalescing cracks can be considered in a section plane as a finger-print of the material's history involving environmental conditions and various loadings (Fig. 1).

At the same time it should be linked up with life expectancy of the present-day material under given conditions. Though ideally a logical concept, the exploration of underlying dependencies is very complicated. In this paper we will limit ourselves to quantitatively describing the damage structure as input for the aforementioned dependencies. The nature of this problem is three dimensional (3D). So, to get access to the relevant 3D information would require a random set of section images. This can be arranged for soft tissues, but it is a laborious and time consuming operation when dealing with a material like concrete. So, most of the investigations in practice are of a 2D nature only, inevitably leading to biased information on the finger-print characteristics.

A method introduced by the authors reduces the efforts involved tremendously [2-4]. It assumes for the most general case a 3D portion of the crack structure mixed with $2 \mathrm{D}$ and $1 \mathrm{D}$ ones. The method is discussed for the practical cases of concrete under prevailing compressive stresses and under prevailing tensile stresses, respectively. Damage characteristics under such conditions can be assessed on a single so-called vertical section (i.e. in the loading direction). A parallel set of such sections can be necessary, of course, to reduce scatter to acceptable proportions. However, the efforts for sawing, grinding, contrast improvement and quantitative 


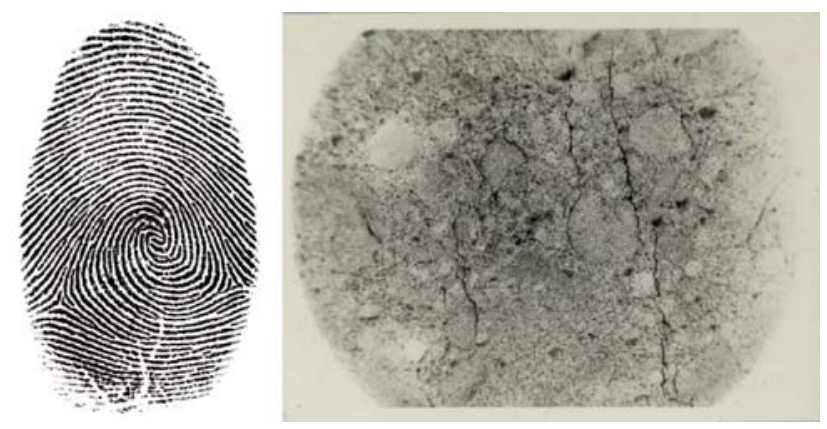

Figure 1: Cracking on micro-level in concrete is predominantly aligned with (vertical) compression direction and mostly runs along particle-matrix interfaces (right); this may be thought of as a type of finger-print (left) of the material under the given conditions.

image analysis are obviously dramatically reduced. The images of such vertical sections can be analysed by the directed secants approach [4-6], whereby the number of intersections is determined per unit of line length for a stepwise rotating grid of parallel lines. Information can be obtained on total crack length $(L)$ per unit of area $(A), L / A=L_{A}$, on the degree of crack orientation, $\omega_{2}$, and on prevailing direction of cracking. This is $2 \mathrm{D}$ information; however, it is readily possible without investment of extra labour to assess the 3D specific crack surface area, $S / V=S_{V}$ , and spatial orientation distribution of the cracks. This will be elaborated for the aforementioned practical cases. Due to the repetitive character of such investigations, one would be tempted to use an automated set up. However, the paper will demonstrate the information generally significantly biased [7-9]. This will be accomplished mathematically as well as by a visualisation method by 'roses' proposed by Underwood [10]. This approach gives detailed insight into the level of bias as a function of the conditions, such as the degree of prevailing orientation in the damage structure.

Damage has fractal properties [11-13]; a phenomenon frequently met in nature (Fig. 2). The lineal features in a plane resort under the category of 'coastline of Britain' phenomena. This implies the total extent of cracking to increase at higher magnification. However, also details of the damage structure change. The forthcoming discussion is therefore limited to an arbitrarily selected level of the microstructure defined by the optical resolution. Information on magnification should be, as a consequence, an integral part of the research report. Fortunately, the type of biases due to digitisation, which will be assessed by comparing the measurements with similar ones obtained on the underlying analog image, are invariant to the optical resolution by which the field under investigation is made. The resolution-dependence requires, nevertheless, that the characteristic measurements by the two different data extraction procedures should be seen in the relative context of (near)-fractality.

Also stochastic heterogeneity in sub-sampling designs, a situation pertinent to experimental approaches in concrete technology, demands specimen sizes and optical magnification to be adjusted to changes in the sizes of the representative volume element [14-16]. These changes are resulting from the variation in technological parameters of which the effects are experimentally investigated. So, the framework for investigating the biases introduced by automation in the image analysis stage is a comparative study that fulfills these requirements [17-19]. The extent of such biases decides on whether the choice for automation in the image analysis stage would be a productive one or not. This problem is explicitly discussed in this paper. 


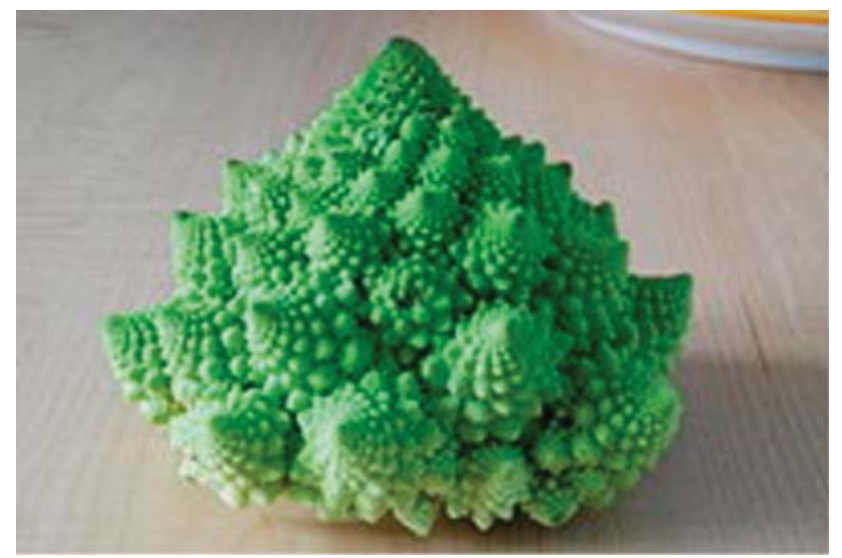

Figure 2: Example of a natural fractal structure (cauliflower) (from Wikipedia).

Last but not least, the image analysis stage is the last one in a multi-stage sampling process, whereby the properties of the concrete in the full-scale structure are the final target of the intersection data in the crack patters. Each sampling stage from the physical element (of the structure), cores, sections, fields to intersection counts is based on limited samples and the average values therefore display scatter. The scatter associated with the prediction of the relevant concrete properties in the full-scale structure, combines the scatter of the various stages. With generally a very limited number of samples in the first and in the second stage because of economy demands, the image analysis stage should not be set up with an excessive number of counts; this does not form a strong impetus for automation, of course [20]. We will see later that the decision not to employ automation can prevent confrontation with significant biases that are generally inherent to automation. However, once aware of this pitfall in the methodology, even the automated design could be set up in such a way that most biases can be avoided.

\section{DAMAGE VISUALISATION}

Various techniques have been developed for the visualisation of damage in the sections or at the surfaces of a specimen. In many cases, the section or the surface is treated by a coloured ink [21], or by a fluorescent spray. Sometimes also impregnation methods are employed by fluorescent dye, or Woods metal that is used as an alternative for Mercury to enter the pores and the cracks under pressure [22]. An interesting full-field method that is basically contact-free and highly sensitive is holographic interferometry (HI) [23]. It is somewhat related to a natural phenomenon that is more widely known, i.e. moiré, as revealed by the tree leaves in Fig. 3.

In the present case, light bundles due to the un-deformed and deformed states of the same body interfere to reveal fringes that reflect equal surface displacements of the stressed body or specimen. Discontinuities in the fringe pattern can therefore be associated with cracks. Hand-copied crack patters are the analog images that form the basis for the quantitative image analysis procedures, as discussed herein. As a sole example of a HI application, Fig. 4 shows a mortar prism with embedded steel strip of which the mortar cover is removed so that the strip is revealed at both surfaces of the prismatic specimen (strip extends therefore over the full width (or thickness) of the prismatic specimen). The HI picture represents the pull-out situation whereby the strip has been de-bonded from the mortar over its full embedment length and cracks are initiated in the mortar at the strip's end. This picture represents a simple case. When HI is applied for crack analysis in a plain cementitious 


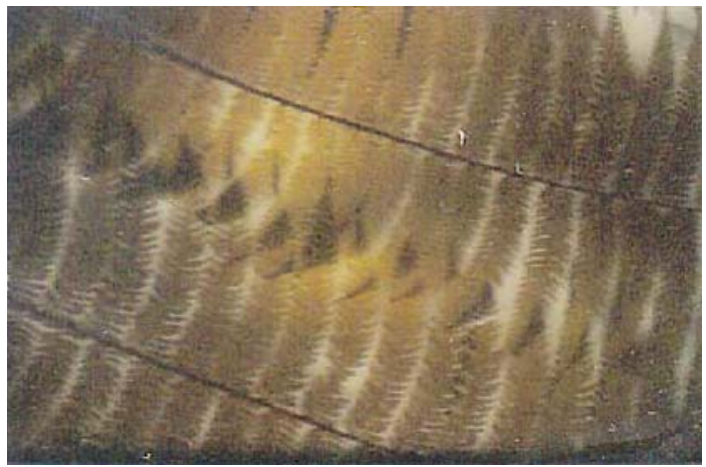

Figure 3: Interference of tree leaves leads in nature to a phenomenon called moiré.

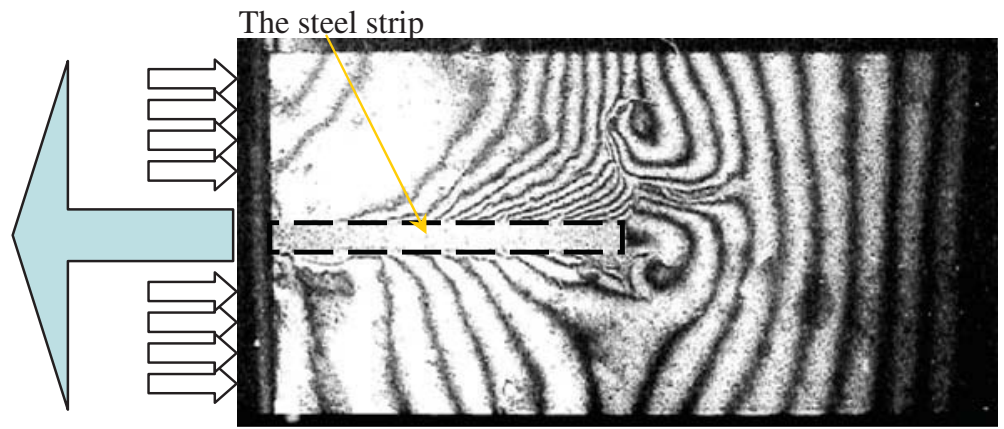

Figure 4: HI picture of cracks at the surface of a prismatic specimen due to complete pull-out de-bonding of a centrally located horizontally embedded steel strip, and of cracks introduced in the mortar. A large arrow indicates the loading of the steel strip that extends to halfway the concrete prism and is embedded over the full thickness of the prism. The smaller arrows indicate the support system during the pull-out experiment.

matrix, pictures are more complicated than in Fig. 4, of course. The images with cracks particularly referred to in this paper are obtained by application of a fluorescent spray at the surface of specimens under load or at section surfaces after unloading. But in all cases, one ends up with patterns of lineal features in the plane that underlie the investigation of the extent of biases in an automated approach to damage assessment.

\section{DAMAGE ASSESSMENT}

The damage structure according to the Stroeven-concept is denoted as a partially linear-planar structure. The 3D portion encompasses the (infinitely) small flat crack element dispersed isotropic uniformly random (IUR) in space. In the 2D portion only the small crack elements are collected that are parallel to an orientation plane, however otherwise they are 'randomly' distributed. The 1D portion comprises the crack elements all parallel to an orientation axis, however otherwise 'randomly' dispersed. When the 2D portion can be neglected, a so-called partially linearly oriented system is obtained. This model can be used in situations where the compressive stresses are prevailing. Alternatively, in dominating tensile stress states the partially planar oriented damage model can be employed, whereby the $1 \mathrm{D}$ portion is neglected. 
Damage can be seen as surfaces distributed in space eventually as a part of a spatial network structure. The two crack surfaces at a very small - so-called crack opening - distance are assumed coinciding in this approach. Crack density is commonly expressed in a total surface area, $S$, per unit of volume, $V$. So, leading descriptor of the damage structure is $S_{V}$ in $\mathrm{mm}^{-1}$. Alternatively, the total crack length, $L$, per unit of area, $A$, yields the $2 \mathrm{D}$ crack extension measure $L_{A}$. Observations are accomplished by superposition of line grids on images, of which Fig. 5 (left) reveals only a small part at moderate magnification. This author has extensively used this method of directed secants in the past 30 years, whereby contrast was improved by applying a fluorescent spray [4, 7, 24-26]. Also other researchers in concrete technology have employed the very method [5, 27-30]. Figure 5 (right) shows the grid orientations on a full-size hand-made copy of a section image of a specimen subjected to direct compression in the vertical direction. Larger aggregate grains are visible as crack-free areas.

Microscope images such as Fig. 5 (left) are obtained under a moderate magnification (10-20x). Figure 5 (right) is a hand-made copy of a camera picture of a $100 \times 200 \mathrm{~mm}$ section. In both cases, photos were realised under illumination by UV light. All cracks of $0.5 \mathrm{~mm}$ and more were selected [7]. So, in both situations the magnification is fixed.

In earlier efforts, we had categorised the cracks as to their length, leading to a probability density function (pdf) for crack length [24, 25]. It suffices here to mention that such pdfs were extremely skew to the left, so revealing a disproportional amount of small cracks. The involved structure-sensitive nature of the crack length would lead to pdfs for larger images (at the same magnification) with shifted mode and mean values; we touch here upon the so-called size effect inherent to sub-sampling designs. For these fundamental aspects of sampling that govern the framework for the problem at issue, the interested reader is referred to the relevant literature [16-18, 31]. Although it forms just the framework, it must be obvious that violations to it will make any effort bestowed on the quantitative image analysis stage useless and so will be the forthcoming discussion on biases introduced by automation.

\subsection{Concrete in compression}

Uniaxial compressive stresses produce predominantly cracks that are parallel to the stress direction. High residual stresses in the aggregate particle-matrix interface may be the result of pre-loading.
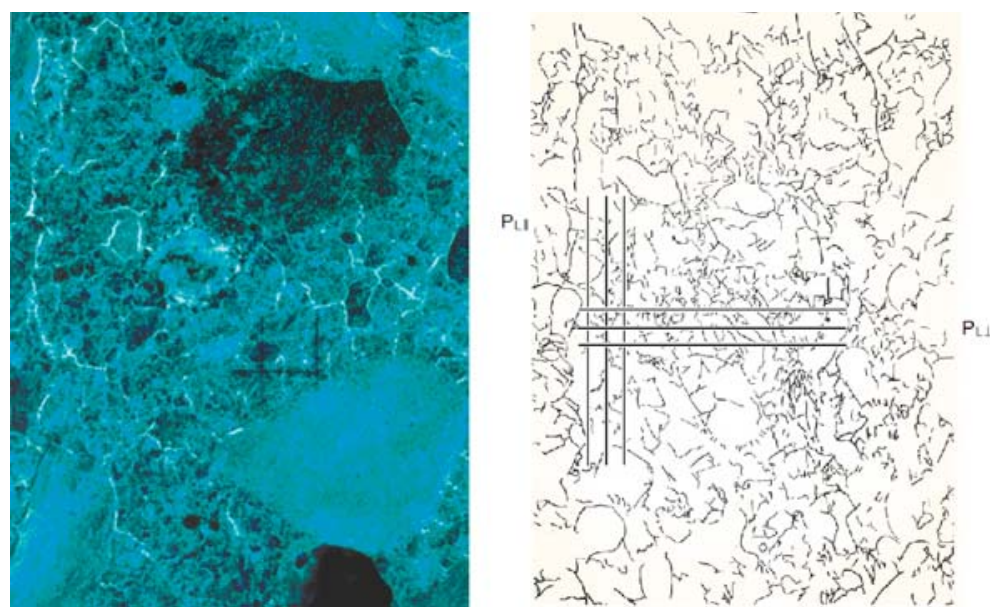

Figure 5: Load-induced cracks in a section plane visualised by fluorescent spray (left), and application of the method of directed secants for the assessment of intersection densities (right). 
Similarly, pre-mature cracking will also arise from volumetric changes resulting from cooling and drying actions. This crack dispersion will have a 'random' character. Upon increase of the compressive loading, gradually further crack propagation along the particle-matrix interfaces and coalescence among such interface cracks through cement pockets will lead to a prevailing crack orientation aligned with the global compressive loading direction.

Particularly at the top of larger particles, tensile crack arrays at interfaces of small sand grains are formed due to high shear stresses, the so-called en échelon crack arrays. Slip along such crack arrays causes a gradual departure of the stress-strain curve from linearity. This point is associated therefore with the discontinuity point or limit of elasticity (Fig. 6). These slip lines cause the prevailing crack direction to rotate. However, upon further load increase the process of crack coalescence through the pocketed cement paste continues; the prevailing axial direction of cracking is regained. So, under relative high uniaxial compressive stresses, prevailing crack orientation will be in the loading direction. This can be assumed an axis of rotational symmetry, as a consequence, and is referred to as the orientation axis of cracking.

Figure 7 shows a cubical part of the investigated concrete. The $z$-axis is the loading direction in compression (or tension), so also constitutes the symmetry axis and thus the orientation axis in compression. In a generalised set up, we assume a portion of the cracks (in fact the small flat element of the cracks) distributed isotropic uniformly random denoted as the $S_{V 3}$ component. The remaining portion consists of the (small flat elements of the) cracks parallel to the orientation axis, denoted as the $S_{V 1}$ component. Total crack density is the summation of both components ( $S_{V 1}+S_{V 3}=S_{V}$ ). The proper approach (in technical as well as economic respect) is sampling by vertical sections. Hence, the core drawn from a reinforced concrete structure (or the specimen made in the laboratory) should be cut to yield one or more image planes parallel to the orientation axis. The $\{x, z\}$ - and $\{y, z\}$-planes in Fig. 7 are vertical sections.

Such section images can provide the 3D information on $S_{V}$. Averaging over more vertical images reduces scatter, and thus improves the reliability of the results. The results are unbiased, which means that averaging over an increasing number of images will bring the average closer and closer to the population value we are interested in. The analysis of the images is accomplished by line scanning. A grid of parallel lines is superimposed on the crack pattern, successively in the direction of the orientation axis
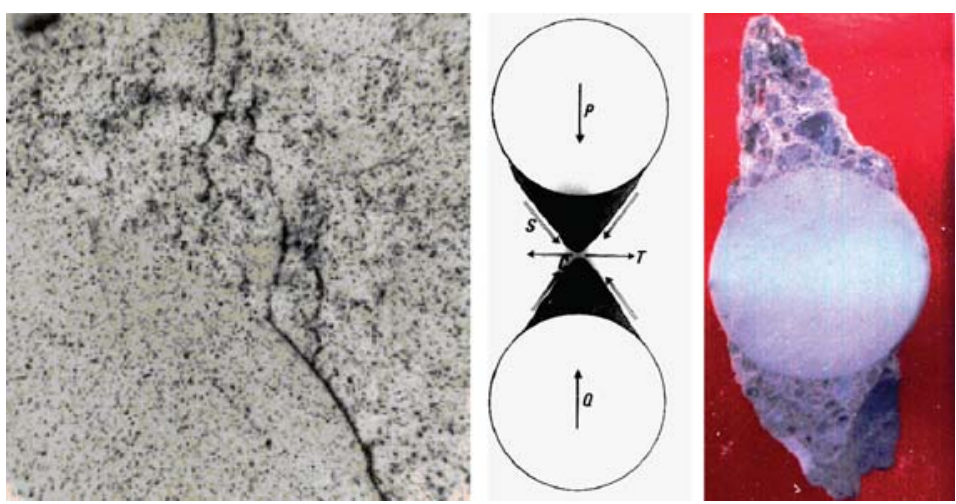

Figure 6: Characteristic discontinuity stage in damage evolution under compressive loading. Left: En échelon crack array. Middle: Slip due to shear forces $S$ along zones weakened by en échelon crack arrays induce vertical cracks as a result of secondary tensile stresses $T$. Right: After fracture, elements (aggregate grain + mortar caps) are regained in the debris [32, 33]. 


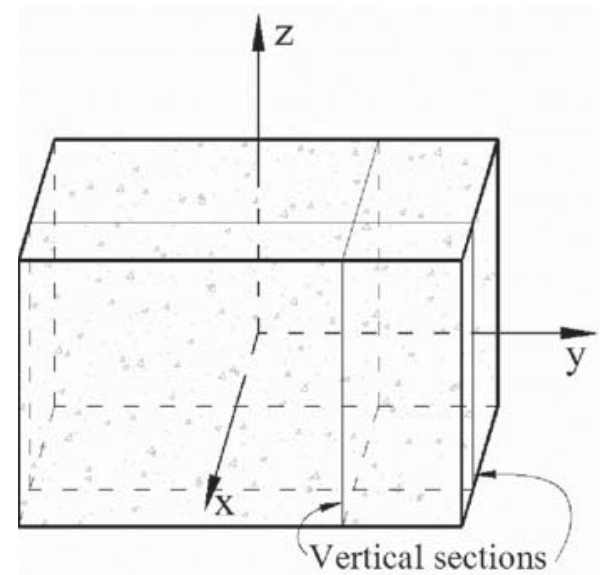

Figure 7: Cubic element of the investigated cracked concrete, subjected to either a tensile or a compressive loading in the $z$-direction. The $1 \mathrm{D}$ linear crack portion in compression is parallel to the $z$-axis (=orientation axis); the 2D planar crack portion in tension is parallel to $\{x, y\}$-plane (=orientation plane). The vertical sections (or planes) in both cases are indicated.

(indicated by index $\|$ ) and perpendicular to it (indicated by index $\perp$ ), as shown Fig. 5 (right). The following relationships can be derived between observations and 3D crack features

$$
P_{L \square}=\frac{1}{2} S_{V 3} \text { and } P_{L \perp}=\frac{1}{2} S_{V 3}+\frac{2}{\pi} S_{V 1}
$$

Hence, the crack density (or the specific crack surface area) is obtained by simple mathematical manipulations, yielding

$$
S_{V}=\frac{\pi}{2} P_{L \perp}+\left(2 \frac{\pi}{2}\right) P_{L \square}
$$

$P$ in eqns (1) and (2) stands for the number of intersections of the grid lines and the cracks. The constants account for probabilities that the cracks appear in the section image and there upon intersect with the directed secants $[6,8-10]$.

\subsection{Concrete in tension}

Damage evolution under direct tensile stresses is a somewhat simpler process than in the compression case. High residual stresses due to pre-loading and due to cooling and shrinkage are released by crack formation of random nature at interfaces between the aggregate grains and the cement paste. At increasing tensile stresses, further crack propagation and crack coalescence through the cement pockets will take place predominantly perpendicular to the tensile stress field. Yet, it is demonstrated in Fig. 8 that this is too simple a concept. Even significantly beyond ultimate tensile stress (UTS) along the descending branch of the stress-strain curve, crack orientation is shown fluctuating in the so-called fracture process zone around the major crack that ultimately will connect the two notches. This major crack path is however still not clearly delineated. The major crack path became obvious after even larger deformations, with an applied stress about 50\% of UTS [7]. Contrary to fracture mechanical modelling approaches, this crack didn't start at a notch but gradually formed in the higher stressed region between the notches due to coalescence of micro-cracks. 


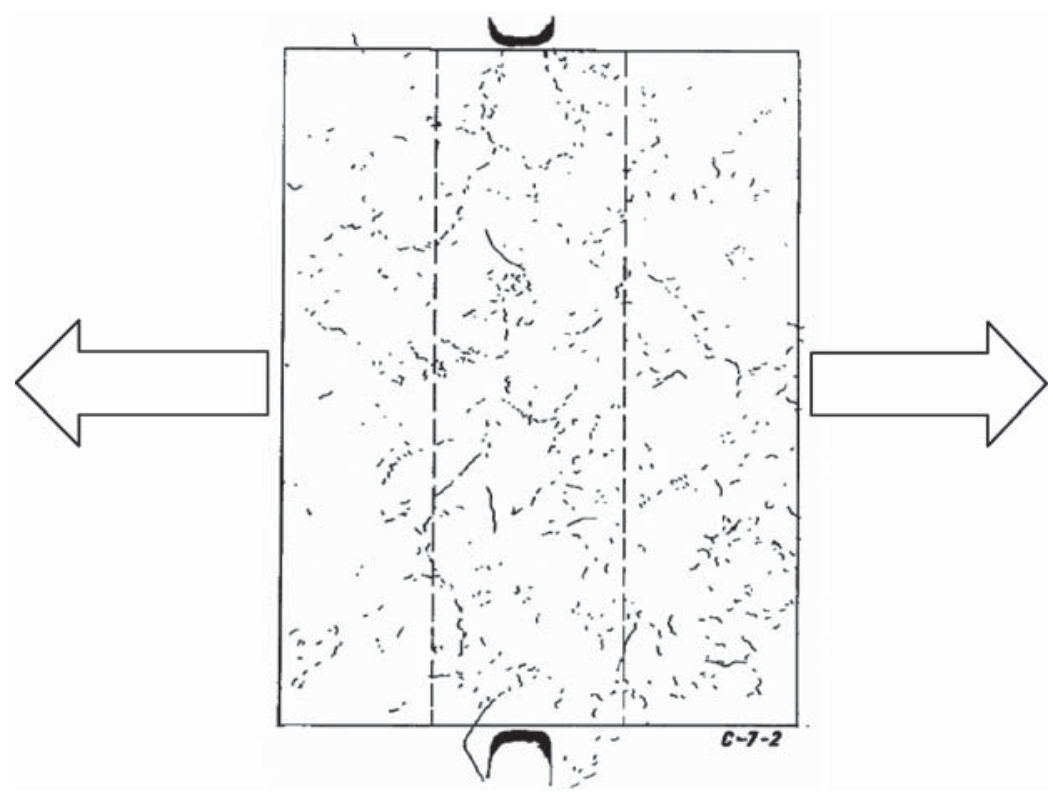

Figure 8: Damage state in vertical section of central part of two-sided notched prismatic concrete specimen (with $50 \mathrm{~mm}$ between the notches visible at top and bottom) subjected to uniaxial tensile stresses (in horizontal direction) along descending branch at about three quarter of UTS. Contrast was improved by a fluorescent spray. For additional experimental details, see Stroeven [7].

The analytical methodology is very similar to the one described for the compression case. The vertical section indicated in Fig. 7 is again parallel to the global tensile stress field. The grid is also successively superimposed in the stress direction and perpendicular to it, with the same indices ( $\|$ and $\perp$, respectively) accounting for the position of the grid. In the present case we have:

$$
P_{L \square}=\frac{1}{2} S_{V 3}+S_{V 2} \text { and } P_{L \perp}=\frac{1}{2} S_{V 3}
$$

Again, simple manipulation will yield:

$$
S_{V}=S_{V 2}+S_{V 3}=P_{L \square}+P_{L \perp}
$$

Here, $S_{V 2}$ stands for the portion of the cracks perpendicular to the tensile stresses, with $S_{V}=S_{V 2}+S_{V 3}$. To accomplish such operations, the contrast should be improved by ink penetration or by application of a fluorescent spray (used in the case of Figs 1 and 5) or dye. Details can be found in the relevant literature, such as in $[4,6,7,25,26]$.

\section{BIASES DUE TO AUTOMATED SET UP}

\subsection{Analog images}

An elegant way to reveal differences in outcomes of quantitative image analysis approaches by directs secants to analog and (four-connexity) digitised images is to make use of the earlier mentioned Stroeven-concept. Note that 'four-connexity' implies a continuous crack trace replaced 
by orthogonal components in a digitised image, as will be shown in what follows. Higher order digitisation techniques require some adaptations.

$L_{A}$ is assumed consisting of two portions, a 'random' one, denoted by $L_{A \mathrm{r}}$, and a fully oriented one, indicated by $L_{A 0}$. The latter 'sticks' (short straight elements as part of the $2 \mathrm{D}$ cracks) run parallel to the orientation axis that supposedly coincides with the positive $x$-axis. This strategy allows dealing with both portions separately. The rose of intersections per unit of grid line length (intersection densities) of the random portion approximates (for very large images) a circle around the origin with radius $P_{L r}$ (Fig. 9 at the right). Note that a 'rose' represents the observed values of the number of intersections per unit of grid line length plotted from the origin in the successive directions of the grid. So, the distance from the origin to the rose's perimeter is proportional to the specific observation. The rose of intersection densities for the oriented portion is a circle through the origin (Fig. 9 at the left). Its main extension is $P_{L o}(\theta=\pi / 2)=P_{L o \max }$, so perpendicular to the orientation direction of the sticks in the oriented portion.

When combined, the rose of intersection densities is obtained for a partially linear structure of lineal features in a plane, shown in Fig. 10. The intersection density in an arbitrary direction is:

$$
P_{L}(\theta)=P_{L x}+P_{L o \max } \sin \theta
$$
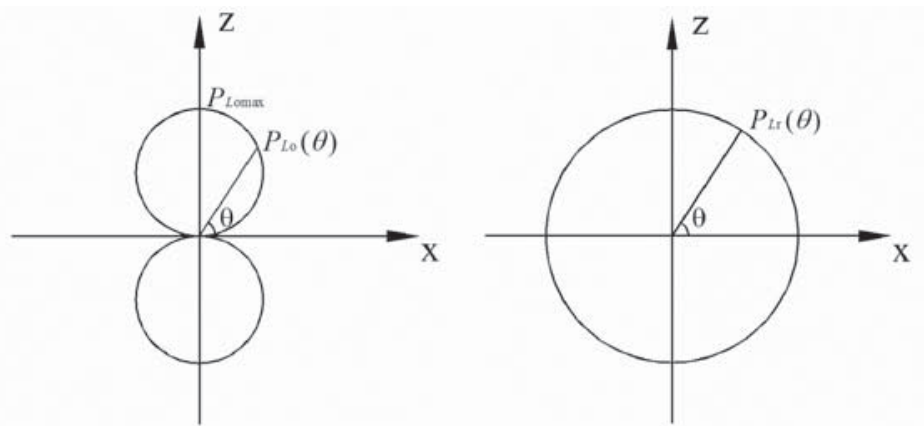

Figure 9: Rose of intersection densities for oriented (left) and random (right) line segments in a plane (together forming the crack pattern in the section plane) for an analog image.

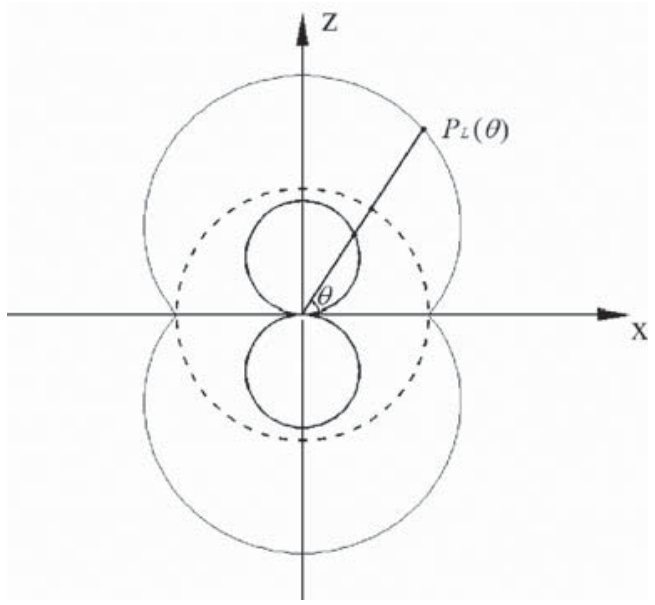

Figure 10: Rose of intersection densities for random (dashed line) plus oriented line segments in a plane (continuous line; small circles) shown in Fig. 9. 
It is readily seen that for $\theta=\pi / 2$ the total rose takes its maximum value, so the cracks are oriented parallel to the $x$-axis. Note that the 2D portion in the image plane can originate from the linear portion in compression or from the planar portion in tension. Hence, both situations are described [6, 8-10].

\subsection{Digitised images}

The smooth contours of the cracks can be conceived in the conventionally digitised images replaced by two orthogonal sets of mono-size sticks as shown in Fig. 11. As before, a distinction can be made between the 'random' portion and the oriented one running parallel to an orientation axis. The random portion consists of two equally large sub-sets of sticks oriented in the respective coordinate directions $\{x, z\}$. This leads to two equally large roses of intersection densities that run through the origin and are orthogonally oriented. Circle diameter is $P_{L r}$. The summation yields a symmetric flower-like rose displayed in Fig. 12 at the bottom. In an arbitrary direction, the intersection density is:

$$
P_{L r}(\theta)=P_{L r}(\sin \theta+\cos \theta)=\sqrt{2 P_{L r}}\left|\cos \left(\theta-\frac{\pi}{4}\right)\right|
$$

A striking but expected observation is the preferred orientation in a direction enclosing an angle $\pi / 4$ with the positive $x$-axis; the random portion is reflected significantly biased by a digitised image with a maximum value of $\sqrt{2} P_{L r}$.

The oriented portion in the digitised image gives rise to a similar rose of intersection densities as in the analog case. Hence, the intersection density in an arbitrary direction $\theta$ is given by:

$$
P_{L}(\theta)=P_{L o \max } \sin \theta+\sqrt{2 P_{L x}}\left|\cos \left(\theta-\frac{\pi}{4}\right)\right|
$$

The direction of the principal axis of the total rose is found for zero value of the first derivative of eqn (7). This leads to $\operatorname{tg} \theta=\left(P_{L x}+P_{L o \max }\right) / P_{L x}$. Hence, for only random cracks, the principal direction of orientation is under $45^{\circ}$ with the positive $x$-axis. For only the oriented portion, we have $\theta=\pi / 2$; cracks run in the $x$-direction. However, for the practical case of partial orientation, the principal direction of orientation will be anywhere between these two values. Hence, results are biased to an unknown degree.

\section{EXAMPLE OF DAMAGE ASSESSMENT}

Figures 13 and 14 present results on the analysis of shrinkage cracking at the free surface of a 40-mm cubic specimen. Obviously, the vertical axis is the orientation axis in the partially linear system because of approximate rotational symmetry. Four serial and thus vertical section images are
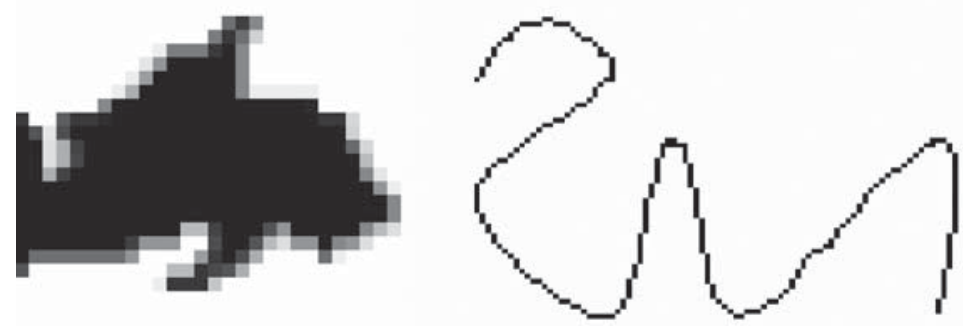

Figure 11: Digitised particle perimeter of dispersed surfaces in 3D space (left). Digitised crack contour revealing a similar structure of orthogonal sticks (right). 


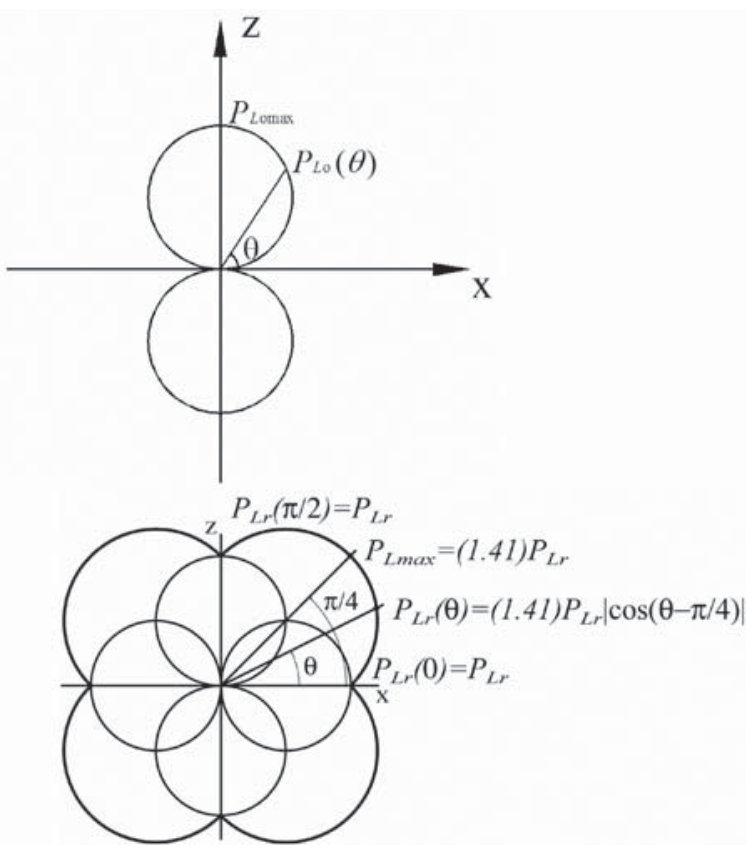

Figure 12: Rose of $P_{L}(\theta)$ values for oriented (top) and random (bottom) line segments in digitised image of crack pattern.

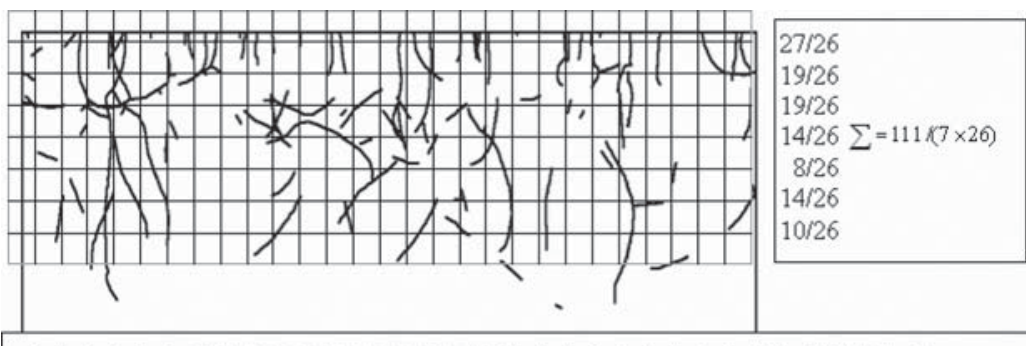

$2126332108333223431112311101 \quad \sum=75 /(27 \times 6)$

Figure 13: An example of intersection counting by an orthogonal grid. Image represents $40 \mathrm{~mm}$ wide mortar section of a cube specimen containing $21 \%$ by volume of $4 \mathrm{~mm}$ glass balls as a maximum aggregate. The image is a superposition of shrinkage crack patterns of four vertical sections.

superimposed [19]. Figure 13 shows the procedure of intersection counting by an orthogonal grid for the determination of the two portions of the specific crack surface area $\left(S_{V 1}\right.$ and $\left.S_{V 3}\right)$. Results can be found in [26].

For the present purpose more relevant, Fig. 14 presents the intersection density observations displayed by the Underwood rose. It reveals a high degree of similarity with Fig. 10, constituting experimental evidence in support of the Stroeven-concept. 


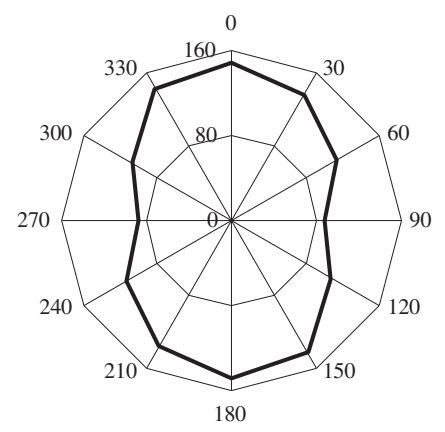

Figure 14: Orientation distribution of crack length in $\mathrm{mm}$ (=rose of intersection densities) of the four superimposed images of the mortar of Fig. 13. For biased radar diagram derived in an automated analysis, see [19].

It should be noted that this problem is more complicated than pursued analytically in this paper, since one is confronted in this illustrative example with a damage gradient from top-to-bottom. Nevertheless, a global representation of the crack orientation distribution by the a rose of intersection densities derived from the digitised image is found in agreement with the global modelling concept. Moreover, the seriously biased radar diagram in [19] constitutes a dramatic evidence of the pitfall of automation, as stressed in this publication.

\section{DISCUSSION}

It should be noted that this paper has limited itself to the simple cases of direct tension and of direct compression, in which the symmetry axis of the damage structure coincided with the $z$-axis. This is guaranteed in laboratory testing, of course. When cores are drawn from the full-scale structure subjected to a complicated stress-strain state, this is in general not so, however. It has been demonstrated in $[8,9,34]$ how under such conditions the spatial value of the prevailing direction of cracking can be assessed. The resulting oblique angle with the $z$-axis constitutes another parameter in the image analysis procedures, which influences the biases by digitisation in a complicated way. The most dramatic situation will arise when the direction of four-connexity digitisation is not adjusted to the $2 \mathrm{D}$ direction of preferred crack orientation in the orthogonal set of section images required for a complete analysis. To determine this direction of cracking, an analog set of images is required! Next, when the digitisation direction is adjusted to the direction of preferred crack orientation, the intersection counts with an orthogonal grid adjusted to the digitisation direction can yield unbiased information, so that 2D and 3D measures for total crack extension can be determined. The orientation distribution of cracking is governed, as discussed herein, by the Stroeven-concept.

\subsection{Crack extent in 2D and 3D}

Total crack length is readily obtained by application of $L_{A}=\pi \bar{P}_{L}(\theta) / 2$. This yields for the analog image (tension and compression):

$$
L_{A}=\frac{\pi}{2} \frac{\int_{0}^{\pi / 2}\left(P_{L x}+P_{L o \max } \sin \theta\right) d \theta}{\int_{0}^{\pi / 2} d \theta}=\frac{\pi}{2} P_{L r}+P_{L o \max }
$$


and for the digitised image:

$$
L_{A}=\frac{\pi}{2} \frac{\int_{0}^{\pi / 2}\left(P_{L x} \sin \theta+P_{L x} \cos \theta+P_{L o \max } \sin \theta\right) d \theta}{\int_{0}^{\pi / 2} d \theta}=2 P_{L r}+P_{L o \max }
$$

Hence, $L_{A}$ as obtained from digitised image is always biased (i.e. overestimated). Only for extremely oriented cracks, the obtained data approximate the correct value. For only random cracks the data overestimate the total crack length by a factor $4 / \pi$. For mixed situations, the bias can be anywhere between 1 (unity) and $4 / \pi$. The origin of the biases is illustrated in Fig. 15. All counting observations by sweeping test line in directions different from the orthogonal ones adjusted to the orientation axis are biased.

For the crack extent in $3 \mathrm{D}$, use can be made of the relationship $S_{V}=2 \bar{P}_{L}$, but data derived from application of sweeping test lines on digitised vertical section images can neither be used for reliably predicting specific crack surface area. So, comparison between $S_{V}$ data derived from analog and digitised images is senseless.

We should stress again, as we argued earlier, that when the direction of preferred orientation of the damage structure can be estimated sufficiently accurate, as assumed herein, the adoption of the Stroeven-concept would also offer a reliable solution for digitised images! This is because structural characteristics like total crack length and specific crack surface area can be exclusively related to the orthogonal observations in vertical sections, as expressed by eqns (1)-(4).

Equations (2) and (4) transform the orthogonal observations made in the 2D image plane of a vertical section to the 3D reality. In the analog and in the digitised image, we find for the tension and compression cases, respectively:

$$
\begin{gathered}
\text { (tension) } P_{L \square}=P_{L r} \text { and } P_{L \perp}=P_{L x}+P_{L o \text { max }} \\
\text { (compression) } P_{L \perp}=P_{L r} \text { and } P_{L \square}=P_{L x}=P_{L o \max }
\end{gathered}
$$

For the tension case this leads according to eqn (4) to:

$$
S_{V}=P_{L \perp}+P_{L \square}=2 P_{L x}+P_{L o \max }
$$

Similarly, according to eqn (2), we find for the compression case:

$$
S_{V}=\frac{\pi}{2} P_{L \perp}+\left(2-\frac{\pi}{2}\right) P_{L \square}=2 P_{L x}+\frac{\pi}{2} P_{L o \max }
$$

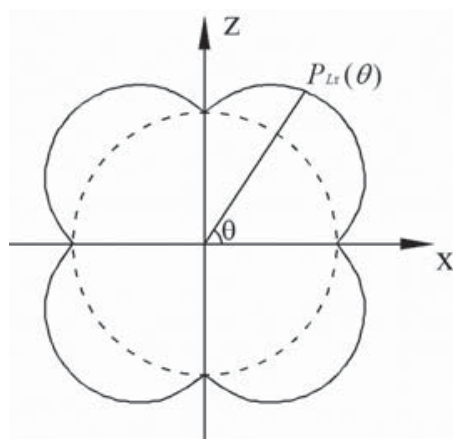

Figure 15: Digitisation-induced biases for random portion of cracks. Dashed line represents the analog image, the solid line the digitised image. 


\subsection{Crack orientation}

When the portions of cracks have been assessed from vertical sections, either on analog or digitised images, the spatial orientation distribution is governed by the Stroeven-concept. The 2D orientation distribution of crack length is as an example given in eqn (8) for the tension case, as illustrated in Fig. 9, and yields:

$$
L_{A}(\theta)=P_{L x}+P_{L o \max } \sin \theta
$$

Similarly, in 3D space for the tension case:

$$
S_{V}(\theta, \beta)=\left(P_{L x}+P_{L o \max } \sin \theta\right) \cos \theta
$$

Executing the averaging operation for the tension case, we find:

$$
S_{V}(\theta, \beta)=2 \frac{\int_{0}^{\pi / 2} \int_{0}^{\pi / 2}\left(P_{L x}+P_{L o \max } \sin \theta\right) \cos \theta d \theta d \beta}{\int_{0}^{\pi / 2} \int_{0}^{\pi / 2} \cos \theta d \theta d \beta}=2 \bar{P}_{L}
$$

yielding the same expression as in eqn (11). Similarly, for the compression case:

$$
S_{V}(\theta, \beta)=2 \frac{\int_{0}^{\pi / 2} \int_{0}^{\pi / 2}\left(P_{L x}+P_{L o \max } \cos \theta\right) \cos \theta d \theta d \beta}{\int_{0}^{\pi / 2} \int_{0}^{\pi / 2} \cos \theta d \theta d \beta}=2 \bar{P}_{L}
$$

providing also the correct expression given in eqn (12).

Note that the cracks in the vertical plane due to the 1D (in the compression case) and 2D portions (in the tension case) are orthogonal, so also the Underwood circles are orthogonally oriented. This leads to the cosine and sine functions ineqns(15) and(16), respectively (i.e. $L_{A}(\theta)=P_{L x}+P_{L o \max } \cos \theta$ for the compression case).

A parameter used to characterise the degree of crack orientation is $\omega$, defined as the ratio of parasitic crack extent over total crack extent. In compression, the linear crack portion is the parasitic one, in tension this is the planar portion. This parameter can be derived for a space system $\left(\omega_{3}\right)$, as well as for a planar one $\left(\omega_{2}\right)$ by the information provided in this paper. As an example, for $\omega_{2}$ this leads to:

$$
\begin{gathered}
\omega_{2}=\frac{P_{L o} .100}{P_{L o}+\frac{\pi}{2} P_{L r}}=\frac{100}{1+\frac{\pi}{2} \frac{P_{L r}}{P_{L o}}}(\%)(\text { analog) } \\
\omega_{2}=\frac{P_{L o}}{P_{L o}+2 P_{L r}}=\frac{1}{1+2 \frac{P_{L r}}{P_{L o}}}(\%)(\text { digitised }) \omega
\end{gathered}
$$

Both parameters decline smoothly from $100 \%$ (only 'oriented' cracking) to $0 \%$ (only 'random' cracking). Still, information obtained from only the orthogonal components presents biased information on the degree of orientation when based on a digitised image.

\section{CONCLUSIONS}

Damage in concrete can be visualised in sections of concrete subjected to loading. Contrast improvement is required. Also, deformations at specimen surfaces can be recorded by holographic 
interferometry, whereby discontinuities can be associated with cracks. In all cases, images will reveal cracks as lineal features in the plane. Such images are conventionally submitted to quantitative image analysis by a sweeping test line system. For the most general case, random sampling (i.e. sectioning!) would be required. In practice, this is limited to orthogonal section images only, however without proper assumptions even this laborious concept would lead to biased information.

For the practical cases of prevailing compressive or tensile loadings, (a set of) vertical sections will do according to the 'Stroeven-concept', restricting dramatically efforts required for preparation of samples and image analysis. Nevertheless, only a comparative study can be performed on the (traditionally) subrepresentative level. Stochastic heterogeneity requirements demand in this comparative study adjustment of image size and magnification to the changes in the representative volume element resulting from variation of technological parameters, the effect of which is pursued in the investigations. The fractal nature of cracking also requires maintaining such a constant relative level of magnification.

The choice to automate the quantitative image analysis stage is a risky one, since characteristic measures for a damage structure, like total crack length (or specific crack surface area) and degree and direction of prevailing crack orientation will be seriously biased due to digitisation. Only when the Stroeven-concept of damage modelling is accepted, and the direction of four-connexity digitisation is adjusted to the prevailing direction of cracking (as realised in the discussed cases for direct compression and tension), the orthogonal intersection counts (i.e. in the loading direction and perpendicular to it) will be unbiased. So, crack extension measures can be assessed in 2D as well as 3D. Orientation distribution is governed by the Stroeven-concept. In manually analyzing cracking in analog images this pitfall can be avoided. Total scatter encountered in a multi-stage sampling scheme that underlies estimates of engineering behaviour on the basis of such crack assessment data does not form a strong impetus for bestowing intensive effort on the image analysis stage as pursued by automation. So, this can better be avoided.

Discussed in this paper is basically a research tool, providing global 3D characteristics of damage. However, when the surface of a concrete structure could be approximately associated with a vertical plane (which depends on the local stress situation, as discussed herein), the surface crack pattern could be analysed as described in this publication. Yet, crack mapping during periodic inspections for health monitoring is quite another problem, because prime interest will be in local crack (groth) characteristics instead of in global ones.

\section{REFERENCES}

[1] Brandzaeg, A., Failure of a Material Composed of Non-isotropic Elements: an Analytical Study with Special Application to Concrete, Det. Kong. Norske Vidensk. Selensk. Skr., 12, 1927.

[2] Stroeven, P. \& Shah, S.P., Use of radiography-image analysis for steel fiber reinforced concrete. Testing and Test Methods of Fiber Cement Composites, ed. R.N. Swamy, Constr. Press: Lancaster, pp. 345-353, 1978.

[3] Stroeven, P. \& de Haan, Y.M., Structural investigations on steel fiber reinforced concrete. High Performance Reinforced Cement Composites, eds. H.W. Reinhardt \& A.E. Naaman, E\&FN Spon: London, pp. 407-418, 1992.

[4] Stroeven, P., Damage evolution in concrete; application of stereology to quantitative image analysis and modeling. Advanced Materials for Future Industries: Needs and Seeds, eds. I. Kimpara, K. Kageyama \& Y. Kagawa, SAMPE: Tokyo, pp. 1436-1443, 1991.

[5] Ringot, E., Automatic quantification of microcracks network by stereo-logical method of total projections in mortars and concrete. Cement and Concrete Research, 18, pp. 35-43, 1988. doi:10.1016/0008-8846(88)90060-9, doi:10.1016/0008-8846(88)90119-6 
[6] Stroeven, P. \& Hu, J., Stereology: historical perspective and applicability to concrete technology. Materials and Structures, 39, pp. 127-135, 2005. doi:10.1617/s11527-005-9031-6

[7] Stroeven, P., Some observations on microcracking in concrete subjected to various loading regimes. Engineering Fracture Mechanics, 35(4/5), pp. 775-782, 1990. doi:10.1016/0013-7944(90)90161-9

[8] Stroeven, P., Stroeven, A.P. \& Dalhuisen, D.H., Image analysis of "natural" concrete samples by automated and manual procedures. Cement and Concrete Composites, 23, pp. 227-236, 2001. doi:10.1016/S0958-9465(00)00064-0

[9] Stroeven, P., Stroeven, A.P., Dalhuisen, D.H. \& van der Meer, J.J.M., Stereological analysis of ice flow-induced preferred orientation of small clasts in Tertiary tillite matrix of Mt. Feather. Acta Stereologica, 18(1), pp. 49-60, 1999.

[10] Underwood, E.E., Quantitative Stereology, Addison-Wesley: Reading (MA), 1970.

[11] Stroeven, P., Fractals and fractography in concrete technology, Brittle Matrix Composites 3, eds. A.M. Brandt \& I.H. Marshall, Elsevier Appl. Sc.: London, pp. 1-10, 1991.

[12] El-Saouma, V.E., Barton, C.C. \& Gamaleldin, N.A., Fractal character-ization of fracture surfaces in concrete. Engineering Fracture Mechanics, 35(1), pp. 47-53, 1990. doi:10.1016/0013-7944(90)90182-G

[13] Carpinteri, A., Fractal nature of materials microstructure and size effects on apparent mechanical properties. Mechanical Matters, 18, pp. 259-266, 1994.

[14] Stroeven, P., Stroeven, M., Size of representative volume element of concrete assessed by quantitative image analysis and computer simulation. Image Analysis and Stereology, 20(Suppl. 1), pp. 216-220, 2001.

[15] Hu, J., Chen, H. \& Stroeven, P., Spatial dispersion of aggregate in concrete; a computer simulation study. Computers and Concrete, 3(5), pp. 301-312, 2005.

[16] Stroeven, P., Hu, J. \& Chen, H.S., Stochastic heterogeneity as fundamental basis for the design and evaluation of experiments. Cement and Concrete Composites, 30, pp. 506-514, 2008. doi:10.1016/j.cemconcomp.2007.12.001

[17] Freudenthal, A.M., The Inelastic Behaviour of Engineering Materials and Structures, Wiley: New York, 1950.

[18] Holliday, L., Geometric Considerations and Phase Relationships, Composite Materials, Elsevier Publishing Co.: Amsterdam, pp. 1-27, 1966.

[19] Bisschop, J. \& van Mier, J.G.M., Effect of aggregates on drying shrinkage micro-cracking in cement-based composites. Materials and Structures, 35(252), pp. 453-461, 2002. doi:10.1007/ BF02483132

[20] Gundersen, H.J. \& Osterby, R., Optimizing sampling efficiency of stereological studies in biology: or 'Do more less well'. Journal of Microscopy, 121, pp. 65-73, 1981.

[21] Hsu, T.C., Slate, F., Sturman, G. \& Winter, G., Microcracking of plain concrete and the shape of the stress-strain curve. Journal of the American Concrete Institute, 60(2), pp. 209-224, 1963.

[22] Nemati, K.M., Generation and Interaction of Compressive Stress-induced Microcracks in Concrete, PhD Thesis, University of California, Berkeley, 1994.

[23] Bień, J., Burakiewicz, A. \& Stroeven, P., Experimental study of steel-to-concrete bond in plain and fibre reinforced concrete. Archives of Civil Engineering, XL(2), pp. 215-241, 1994.

[24] Reinhardt, H.W., Stroeven, P., den Uijl, J.A., Kooistra, T.R. \& Vrencken, J.H.A.M., Einfluss von Schwingbreite, Belastungshöhe und Frequenz auf die Schwingfestigkeit von Beton bei niedrigen Bruchlastwechselzahlen. Betonwerk Fertigteil-Technik, 44, pp. 498-503, 1978.

[25] Stroeven, P., Geometric probability approach to the examination of micro-cracking in plain concrete. Journal of Materials Science, 14, pp. 1141-1151, 1979. doi:10.1007/BF00561298 
[26] Stroeven, P. \& Hu, J., Gradient structures in cementitious materials. Cement and Concrete Composites, 29, pp. 313-323, 2007. doi:10.1016/j.cemconcomp.2006.10.002

[27] Chaix, J.M. \& Grillon, F., On the rose of direction measurements on the discrete grid of an automatic image analyser. Journal of Microscopy, 184, pp. 208-213, 1996. doi:10.1046/ j.1365-2818.1996.1190672.x

[28] Stang, H., Mobasher, B. \& Shah, S.P., Quantitative damage characteri-zation in polypropylene fibre reinforced concrete. Cement and Concrete Research, 20, pp. 540-558, 1990. doi:10.1016/0008-8846(90)90098-I

[29] Nemati, K.M. \& Stroeven, P., Stereological analysis of micro-mechanical behaviour of concrete. Materials and Structures, 34, pp. 486-494, 2000. doi:10.1617/13604, doi:10.1007/BF02486497

[30] Carcassès, M., Ollivier, J.P. \& Ringot, E., Analysis of microcracking in concrete. Acta Stereologica, 8(2), pp. 307-312, 1989.

[31] Stroeven, P., Impact of materials science and stereology on the design of experiments in concrete technology. International Journal of Microstructure Properties, 4(2), pp. 250-264, 2009. doi:10.1504/IJMMP.2009.028637

[32] Stroeven, P., Damage evolution in compressed concrete. Proc. Int. Conf on Fracture, ed. A. Carpinteri, Univ. Turin: Turin, 2005 (on CD).

[33] Stroeven, P., Some Aspects of the Micromechanics of Concrete, PhD Thesis, Delft Univ. Technology, Delft, 1973.

[34] Stroeven, A.P., Stroeven, P. \& van der Meer, J.J.M., Microfabric analysis by manual and automated stereological procedures: a methodological approach to Antarctic tillite. Sedimentology, 52, pp. 219-233, 2005. doi:10.1111/j.1365-3091.2004.00690.x 